\title{
EDGE AND FOG COMPUTING IN HEALTHCARE - A REVIEW
}

\author{
SUJATA DASH* SITANATH BISWAS, DEBAJIT BANERJEE‡; AND ATTA-UR-RAHMAN§
}

\begin{abstract}
The architectural framework of Fog and edge computing reveals that the network components which lie between the cloud and devices computes application oriented operations. In this paper, an in-depth review of fog and mist computing in the area of health care informatics is analyzed, classified, and discussed various applications cited in the literature. For that purpose, applications are classified into different categories and a list of application-oriented tasks that can be handled by fog and edge computing are enlisted. It is further added that on which layer of the network system such fog and edge computing tasks can be computed and trade-offs with respect to requirements relevant to healthcare are provided. The review undertaken in this paper focuses on three important areas: firstly, the enormous amount of computing tasks of healthcare system can take mileage of these two computing principles; secondly, the limitation of wireless devices can be overcome by having higher network tiers which can execute tasks and aggregate the data; and thirdly, privacy concerns and dependability prevent computation tasks to completely move to the cloud. Another area which has been considered in the study is how Edge and Fog computing can make the security algorithms more efficient. The findings of the study provide evidence of the need for a logical and consistent approach towards fog and mist computing in healthcare system.
\end{abstract}

Key words: Fog computing, edge computing, healthcare system

AMS subject classifications. $68 \mathrm{M} 14,92 \mathrm{C} 42$

1. introduction. The up-coming developments and research in the technology has adjusted the epicenter of the medical sciences not only for analysis but rather how to prevent those diseases with incomplete recognition and appropriate along with precise health information through a leading in demand technology called the "Internet of Things".

In order to record physiological symptoms and signals, various efficient devices have been discovered and are feasible in the market which can be easily connected to the internet over smart phones, computer or any nodal devices. There is a high potential appeal of the fog computing inside the IoT-based monitoring systems as per the contemporary surveys. The Internet of Things is pointed out towards the physical objects like appliances, electronics, devices which are always developing throughout the world that can feature internet connectivity within an IP address using unique identities are interconnected for enabling the various objects to interact and act accordingly. For obtaining various essential signs within real time, efficient and working in active way occurring in medical devices which includes ECG or for maybe for measuring pressure and temperature, WBAN is used as among various basic prototypes in IoT methodology of healthcare. WBAN establishes a multitude imperceptible or attire sensor node to percept and convey the data over a wireless network. The transmission protocols used for this are Wi-Fi or IEEE.802.15.4. Basically WBAN-based structure is Low-cost and power competent and plays a leading role in several region of healthcare environments such as tracking, and care clinic towards various disease management including chronic and taken precaution towards it. Distant cloud servers are used in many health monitoring systems for keeping and organizing huge data possessed as of sensor nodes in huge quantity. Cloud computing has a number of benefits like services which are made cheap, capacity for the huge facts storing capability and more surplus preservation rate but few challenges also exists like large data transmission, location perception and latency-sensitive issues. For the slowdown in data transmission and data error packet dropping possibilities have increased. More the data is sent over a grid system, the greater is the chance of mistakes that may result in wrong or less precise treatment decisions for which there will be adverse effect on emergency situation and critical illness of mankind. Thus, there will be a great requirement of minimizing the flow of data absorbed system in excess with the value of provision. One solution for accomplishing the problem of a predictable gateway and a remote cloud server is by adding a coat in between them. This added coating is termed as fog sheet and it aids in lowering the large chunks data but also yet guarantying service quality and so protecting the bandwidth of the network by pre-analysing the

\footnotetext{
*North Orissa University, Baripada, India (sujata238dash@gmail.com)

${ }^{\dagger}$ North Orissa University, Baripada, India (sitanathbiswas2006@gmail.com)

${ }^{\ddagger}$ KIIT University, Orissa, India (debajit2409@gmail.com)

$\S$ Imam Abdulrahman Bin Faisal University, Saudi Arabia (aaurrahman@iau.edu.sa)
} 
information. Also, fog computing provides services of higher level at the edge of the wireless systems well as reduces the burden of the cloud [1]. Although the cloud computing hypothesis is brought by cloud computing at the edge of the network, it also reports which aren't proved before unfit in the cloud computing paradigm. Some of the fundamental characteristics of fog computing are low latency, geographical distribution, edge localization, location awareness, inter operability, as well assist aimed at on-line analytic. That is why Fog computing can be taken into consideration for advancing the human health observing WBAN-based systems for the feature like low energy, little bandwidth, truncated processing power and involving the hardware with constrained nodes. To say in literal words, the arrangement of WBAN-based system, with cloud computing and also fog computing can be a defensible clarification aimed at summoning in the recent IoT healthcare systems. As a concept,effective IoT-enabled healthcare system constructions presented and can be profited starting the idea of fog and edge computing. Utilizing the arrangement, we all came-up with an effective fog computing and edge computing healthcare systems which will be IoT enabled and can be utilized in bandwidth also with QoS guarantee, and backup reminder. This paper primarily focuses on the following three important aspects:

- The requirements of IoT based Healthcare model and the description of services provided by Fog computing layer to address them.

- The architecture of an IoT based healthcare system embedding Fog computing layer.

- Implementation of the fog computing layer services along with performance and advantages.

2. Related work. There have already been many attempts in creating brilliant access towards healthcare function.Like for example, Chen et al. [18] introduced a technology of wireless sensor network flow which is used for a smart gateway aimed at the health care system. The endorsed gateway operating for instance can over pass amongst public communication system and a wireless sensor network. It basically has some exceptional features like having a data with agreement system, very flimsy database plus the usefulness just before declaring the needy persons in case of any tough times. In addition, the gateway generates the request- response message function which creates a process of shrinking a distant server's fatigue. Mohapatra et al. proposed semi-hybrid architecture by using a sensor cloud which is needed in distant patient monitoring with working network flow [19]. Advantages were taken by utilizing the sensor cloud for patient's health condition which can be monitored and can be shown in their proposed system. The writers did present a cloud computing with a solution intended for patient's data collection in healthcare organizations. Sensors are used in various systems which are adhered to medical equipment's for assembling patient data then sent the data to cloud to provide restricted permit. Yang et al. presented health monitoring gateway system based on Smart phones [21]. The recommended requirements for a gateway in a Bluetooth console to upload gathered data and send them to remote servers. In [22], the sensing servers are used as gateways in the system which is handled by the sensor network system. But, the proposal is exaggerated the insufficient and extensible as well as inefficient for many applications. In many organizations [23], authors have researched and proposed an idea with an example of a lively IPv6. Less powered wireless with area network $(6 \mathrm{LoWPAN})$ is a signal router which was established on Hidden Markov Model. This model was used for developing settlement in the health status. In [24], the researchers have already designed a mobile gateway for pervasive healthcare system used for ZigBee and Bluetooth. The gateway which favours the feebleness with many services such as investigation of the medical data etc. are efficient, though the gateway can be ineffective within terms of actual power utilization which cannot be recommended for practical use. Zhong et al. presented an alternative way centered on a mobile phone which is used to connect sensor network system nodes along with the devices promoting CDMA or Bluetooth [25]. In a relative work [26], the recommended design can achieve data through many individual health gadgets. USB and ZigBee along with Bluetooth can be considered as a communication process. Subject including body area sensor network systems can be used to attract the attention among a lot of researchers during the past few years. Specifically, in the healthcare domain numerous amount of work has exponentially expanded various undiscovered stepping stones with only one intention for betterment of healthcare monitoring system.

As few works seek the newer systems along with further methods besides number of services although some other researchers approached to suggest new way or else new approaches. Still, some narration in the aboveexplained examples, a great quantity of systems are specifying along with ZigBee though it can be tough for guarantying the characteristics of service done in ZigBee while observing streamed bio-signals like ECG, EMG etc, with the extreme data rate nearly at $250 \mathrm{kbps}$ of ZigBee. Oppositely, Bluetooth Technology can be used to 
solve problems of ZigBee which is lesser data speed and other small-range communication protocol. In spite of this, maybe it is tough for depicting a gateway for supporting mobility as well as employing data through multiple targets with utilizing Bluetooth Technology. The above systems fundamentally use simple gateways from node so collect data and transmit these data for remote servers. Further, sometimes any of these workings had been accounted completely taking advantages of the computing paradigm about fog and contributing insightful to the gateways. The target of the section is used to uplift the IoT-based health monitoring system utilized among different surroundings like among family also in hospital just by imparting the smart gateway [27]along with fog layer consists an extra advanced services. Fog computing conception is actually the elaboration of pointing applications from the cloud computing paradigm along with a vision and province where the full support of the prototype from the cloud is not achieved. Few among them includes fast response employed containing video conference applications, besides Voice over Internet Protocol (VoIP) which requires very less latency for the reason that QoS might get reduced by unusual delays.

- An Enormous data solicitation which is assembled in a huge load with data taken from countless sensors after which it transmits the data via the networks essential for the availability to achieve high bandwidth $[28]$.

- Observing the applications, which can be controlled constantly, needs no interruption during data connection because of connectivity loss takes place among the cloud then monitoring systems [29]. Features use din the presentations are mentioned above which were indistinguishable along with the features of health monitoring systems in real-time. These network that have massive quantity of collected data commencing a variety of environmental along with bio sensors. Later this massive volume of data transmits above the network which is ultimately monitored remotely through end users like care-taker or doctors. Hence, fog computing stands on these systems effectively and is appraisable that instead of removing before lessening the importance of the cloud in IoT applications, from the facet of location awareness,less latency, scalability, real-time interactions, heterogeneity and inter operability that fog computing technique is completely cooperated as well as compatible by the cloud to enhance the existing IoT applications, rather than replacing or lessening the importance of the cloud in IoT applications. [30].

3. Fog Computing. Fog computing, networking, storage and other domain with particular services are handed over to the IOT system by Fog computing layer. The Healthcare domain differentiates it from the other Iot applications by having the most used feature of remote monitoring which require high degree of reliability. The protection and personal aspects towards the health care were highly essential and should be implemented along with the Fog layer focussing on the health care. Moreover,with that it permits the Healthcare IOT to address the necessities of the proximity of Body Area Network (BAN) to Fog layer. Some necessities along with the Healthcare IOT services were inclusive and useful for any application domain. The concluding view of the Fog layer and services are discussed separately and shown pictorially in Figure 3.1.

3.1. Managing Data. Data management is important in Fog computing because the sensory data is locally used in order for withdrawing essential information of anyone to provide a response to user and notify the system plan adaptations. Agreeing to the network architectonics, Fog layer dynamically accepts some huge quantity of data which is auricular from the sensor network system in a very small interval in time, so its main focus is to control the incoming data in order to give a fast reaction with respect to number of users as well as conditions required in the system. This task acquires more importance in healthcare scenarios since latency and uneasiness occurs in decision making might cause incurable damages to the patients. According to the numerous fulfilment of the data management work, we establish it with five different units and all are required for an intelligent e-health gateway. All these parts are named as local storage, data analysis, data filtering, data confining and data fusion.

3.1.1. Local Repository. The gateway requires storing the data which are received from many different sources in a local storage unit so that it can nonchalantly use for analysis in future. The operating system in the gateway contains a file server and a database which stores and also recovers the data. The gateway of the local storage stores the files which are encrypted or maybe contained in a compressed format depending on the type, size, along with the priority of the data. The gateway cause the local storage for exporting data to medical level information like Health Level Seven (HL7)[2][35] when needed. Other features of the gateway 


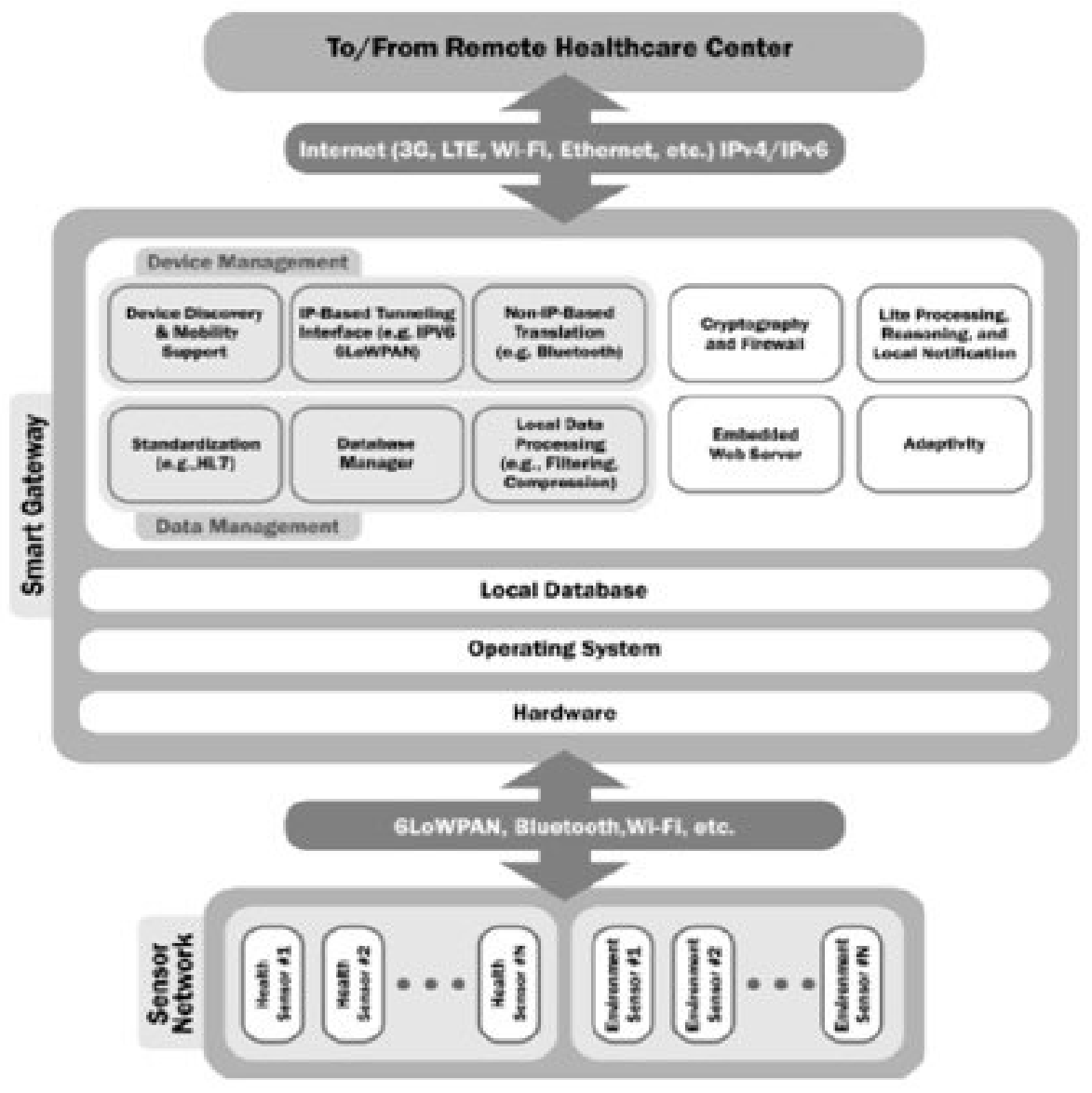

Fig. 3.1. Different Fog layer and services.

like analysing the data, compressing it,filtering, and inscription are basically inter-dependent on a local storage. The overall data rate can be moved among the cloud layer and also the Fog layer depending on the network pace then more towards local estimation along the gateway is visited throughout its power of processing. So it can occur in any displacements during transfer time along with computation time, the local storage acts as a local cache memory to make the data flow reliable. Local storage can also support and save the data when the internet connection isn't accessible among gateway and cloud server. When the internet is restored, the gateways can send saved data into the cloud.

3.1.2. Data Filtration. This is the first step of data processing unit that implements data filtering methods at the edge after receiving it from the sensor grid system. To get a patient's medical condition, numerous bio-signals are collected using pertinent fact-finding from devices like electrocardiogram (ECG), electromyography (EMG) and photo plethysmogram (PPG). These bio-signals relatively include difficult shapes along with tiny amplitude (i.e., millivolts) and accordingly they were dominated by specific unwanted an unreliable sounds which is misled throughout the health monitoring. The noises that are perceived can be thermal noise, electromagnetic interference noise etc. Some available light-weight filters at the nodes of the sensors reduce the assembled noises yet; this may effect in some practical aspects. Thus, the Fog layer containing the data filtering unit which removes the noise and can boost some features of the signals (e.g., signal-to-noise ratio) by the use of different filters (e.g., finite impulse response (FIR) filter) earlier than any other local data analysis.

3.1.3. Compressing Data. For decreasing a massive quantity of transferred data throughout the communication network, data could be flattened by lossless or lossy compression methods used. The lost data 
can generate inapt or incomplete disease diagnosis in healthcare IoT applications because lossless compression is preferred. Even if compression algorithms which are lossless can retrieve indigenous data with precision, are considered to be complex. Also, the algorithms are not most suited for sensor nodes which are actually resource-constrained concerning computation, memory capacity also battery capacity. Like for an instance, in all sorts of sensors lossless ECG compression methods [3, 4, and 5] cannot be used. In particular cases, at sensor nodes these lossless algorithms can be fortunately employed within it but these can also originate huge power utilization and latency. Fog computing aids to improve these defaults of the sensors and can be kept away by diverting all toughes of the sensor nodes towards the gateway and can manipulate these complications effectively during considerations of the real-time circumstances.

3.1.4. Data Blending. It is a data processing unit which can place sensory data commencing the numerous sources for getting more meaningful and strong information. By avoiding repetitive data and restoring the latest data this processing unit can completely diminish the volume of sensory facts. The local data analysis and data transmission gets boosted by the data reduction towards the distant servers. Three separate classes are categorised in Data fusions interdependent concerted also competitive with others [6]. Firstly, At least two different details acquired for more extensive knowledge of the Fog layer achieved from dissimilar places when it mixes with data fusion. For instance, when an ecosystem context data mixes with patient's health specification it shows a more complete data about the patient's state. Secondly,by integrating data which is formulated from one source with (at least) two other sensors the fierce data binding can regulate the quality of the data along with system's decision making capability. For example, utilizing the values taken from an ECG signal also from a respiration signal further powerful heart rate value is extracted. Lastly, using multiple sensors latest information can be yielded with relevant data fusion from a particular place. An example of a cooperative data fusion is the patient's medical state which uses vital signs (e.g., heart rate and respiration rate).

3.1.5. Analysing Data. In data analysing unit the sensory local data is carried out by the healthcare system. The quality of the system in this unit and then sent to the cloud servers after diminishing the response latency and data transmission. For example, when an emergency occurs like patient's health deprivation the emergency matter gets triggered and response comes to play quickly and appropriately because in the cloud instead of getting imparted the local processed data waits for a suitable response. Furthermore, data reliability as well as the consistency of the system is enhanced by the data analysis unit.Finite bandwidth along with Connectivity losses during data transportation in the long-term remote health monitoring were like an inevitable event because the patient may engage in different activities in assorted habitat. Hence,the system's local functionality,sensory data as well as the arithmetic can be directed and taken care by the confined edge of the storage data analysis and also balance the Fog layer after recounting it to the network with a remote cloud.

3.2. Managing Event. A number of major events like change in vital signs, activities or surroundings of the patient can take place in patient's audit. In actualize systems with a specified gateway or maybe a shift in a learned behaviour can be activated by these events. In case of critical condition low latency clarification which might help in notifying health experts, caretaker and even patients are supplied by Fog computing technique immediately. The event administration service fortifies on time and with proper signal delivery in case of medical actions or automatic system actuation when there is a need in quick response. In some medical events like changing the frequency of nerves stimulation related to heart rate or automatic insulin pump with blood glucose level which is regulated with the quick response of activators when it is essential. Some requirements are alerted to the rapid responding team, caretakers or family members of the patient when there is an incident occurred.

3.3. Resource Effectiveness. Resource proficiency is among the most important factors in healthcare IoT function, since catastrophe can lead to severe corollary for sensor nodes' failure to incorrect diagnosis among diseases in resource management. Expectations of collected data presented at end-user's terminals essential to be constantly kept a note of with the energy exhaustion of sensor nodes. They are dealt with in details as following:

3.3.1. Efficient use of energy of the nodes. Sensor nodes in health observing systems should be capable to work for a long interval like a whole day or even a few days which are generally small and resource- 
inhibited, such as miniature battery capacity. Sensor nodes should be functioning efficiently in relation to energy utilization for fulfilling these necessities. For the fulfilment of these tasks many methods contain software and hardware-based procedures which are useful. For example,the design of sensor node hardware is used for specific purposes instead of generic tasks. The unnecessary mechanisms or high-power overwhelming components can be avoided by controlling the energy depletion by this technique. However, customizing the software successively at the nodes is more stimulating for scheming energy efficient nodes. Specifically, the software is simple in order to reduce reckoning time and must be able to execute primary errands of the sensor nodes. For example, sensor nodes in IoT-based plunge recognition systems could intricate the fall detection algorithms by only collecting digital data and address the data to Fog layer [7]. Dramatically condensation can occur in the sensor nodes' power depletion.

3.3.2. Latency. Latency produces unsuitable disease enquiry and delays the supervisory procedure in health monitoring solicitations. Handling and conveying of data both presented latency in IoT for transmitting latency to end-users from sensor nodes via gateways, like Cloud and processing access. In multiple instances,trade-off relationship is often dealing out and showing latency expectancy. However,reduction of the total latency is not always occurred buttes ting the handling data latency. Data processing can even surge the total latency in some circumstances. For some certain sensor nodes and applications a pragmatic differentiable procedure must be done to minimize the total latency and fulfil the time necessities of real-time health monitoring. In other cases, to remove noise and void data some cleaning methods are used in reducing the latency in total as well as huge amount of data transmission. For example,Fog can act as an alternative for sensor nodes to reduce the total latency in an ECG feature withdrawal solicitation; the feature extraction algorithm must be routed towards it $[9]$.

3.4. Management of System. Device supervising consist many parts of Internet of Things organization. In this part, the main area of the debate is on device supervising from the fact of observation of locating device and keeping the connection through in mobility.

3.4.1. Mobility and locating. In the sensor and actuator systems the reserve restriction of devices had been stated previously. The life time of the battery is very important specifically fora battery motorized devices which also require actual supervision. Devices must be in sleep mode in a supervised manner whenever they are about to gain idle condition. Any transmission that takes place when the device is in the sleep condition requires care for the cover of Fog. The patient, who is trying medical sensors for moving from one location to other in a healthcare scenario, can adapt the liable gateway that handles transmission. This shows that the sleeping sensors need to wake up at the sector of various gateways. To locate and effectively handle the sleeping cycle device discovery service must support other devices that can link to a sleeping sensor node; the full utilization can be found for this service [10]. The Fog layer service is used to communicate the nearby kept data from one gateway to other and let it occur without riveting much of the finite resources but also should afford the liberty of flexibility for the patient. For smoothly pursuing the observation activity at the new place in other gateway the device must be located in a new situation, the bestowal takes place. A shortened execution of flexibility and locating device functioning simultaneously is represented in Fog computing [11], These services can be used for more provinces as well as these facilities too.

3.4.2. Inter-operability. The Io $T$ is generally a bunch of diverse set of transmission protocols, platforms and data setups. There are multiple regularity try to initiate constantly amongst the various modules. The present upright subdivisions of presentations require to be joined to simplify the modeling of not just a part of but the entire of the healthcare applications. Traditionally affinity is facing the dare related to resource restriction of the most of the end gadgets. The presence of fog figuring layer is to cover end devices and thus prosper inter operability which caters a vital part in giving amenities gripping. These utilities actually work as a connector among various transmission protocols, data setups plus platforms. At the Fog layer some work can be experienced forgiving semantic inter operability to the data obtained via the sensor son the cloud.

3.5. Personification. Various presentations of Fog computing can be setup in trial or through in run time by behaviour of a machine. This might not bead equate for healthcare structure since users may have hold in various triggers through assorted surroundings and innumerable medical state. So, a powerful plan for the 
machine is necessary, not only to customize the machine performance in granting to specific user requirements but also assertive habituation to the machine over time, exceptionally in case of a crisis. In this regard, it optimizes the energy proficiency and also enhances the local decision making. Various health applications using rule-based skills and machine learning processes are illustrated by Customized scheme behaviour. According to the patient's condition, the proper principles are chosen from the sensor sampling frequency and data transmission rate which are well described. Further, precedence is customized with respect to the medical history of the patient. Simply putting together, the machine would acquire to give more proper heart associated frameworks only if a heart failure is diagnosed for a patient throughout the observation.

3.6. Privacy and Security. Overall, safekeeping is very vital for every solicitation in healthcare is important because a single apprehensive part of a machine might endanger a human life. Fore.g, an insulin pump in an IoT glucose controlling machine can be hacked within 100 feet which is reason for harm [11]. The full machine which consists of sensor nodes, gateways, Fog and Cloud must be protected adequately for delivering a secure IoT healthcare machine. The complete system can easily be manipulated by hackers if somehow any components are hacked. For facts encryption and decryption various like CMAC-AES-128 or AES-128 are used at sensor nodes and gateways, respectively. For constructing IP tables giving consent to proper transmission ports IP table provided by Linux can be utilized in the gateways [12]. For securing the whole machine these techniques can't be trusted as powerful one because these can modify safety at different levels, whereas, in many occasions, it provides a high level of security in the literature [13, 14, and 15]. Still, their complicated cryptographic procedures are not concern to resource-constrained sensor nodes as well as IoT machines. Rahimi et al. $[16,17]$ currently launched end-to-end secure parameters for directing the safekeeping troubles in IoT healthcare machines. The chief parts, comprising of various complicated security algorithms, are executed at the Fog layer as well as the parameters that can give better verification and approval for Health-Internet of Things machine.

4. Edge Computing. Edge computing says about an astonishing eyes opening technology that allow calculating at the extremity of the linkage, on data of downstream or upstream kind. Downstream will come from cloud services where-as upstream data is organized on the part of IoT services. And now we declare "edge" as figuring and integrating grid system locality along the way among data obtain ability and cloud data servers. Like, a smart phone is the border or edge connecting living beings and cloud data, a gateway can be referred to a smart home. The hypothesis of edge computing is that computation should always take place near a data source. According to our idea of research, fog computing can be replaced by edge computing [32] but fog computing marks extra on the substructure side, whereas edge computing stresses mainly on the effective side. We envisage that edge computing when compared with the cloud computing can possess a large and better effect on our community. Fig. 4.1 displays the two-way computing rush for edge computing. Edge computing models, things that acts as data consumers, but can also act as data producers. On the edge, representing the computing works which is commenced on the cloud but also the possessions,can't appeal facility and content from the cloud. Edge computing can conduct data transfer, storing the data, accumulating and handling, as well as spread appeal and delivering amenity from cloud to user. The edge itself requires to be well planned to converge the need that can be effective with praise to reliability, security, and isolation protection with all the kinds of tasks in the network.

In edge computing, the center of attention is on setting the computing attached to the closeness of data causes. This has various advantages contrast to conventional cloud-form computing paradigm. To illustrate the probable advantage we use several premature outcomes from the circle. The response time has moved the computation from cloud to the edge by reducing it from 900 to $169 \mathrm{Ms}$ and the Research workers raised a proof-of-concept platform to execute face acknowledgment presentation [64]. Ha et al. [33] used the cloudlets for offloading the computing tasks for wearable cognitive support, and the advancement of reaction time is between 80 and $200 \mathrm{~ms}$ when the results get displayed. Furthermore, by use of cloudlet offloading the energy utilization could also be condensed by 30\%-40\%. Between the mobile and the cloud with their prototype which could shrink 20 times the executing time and energy for tested uses can perform merge dividing, relocation with merging, and the on-demand instantiation in the clone cloud. 


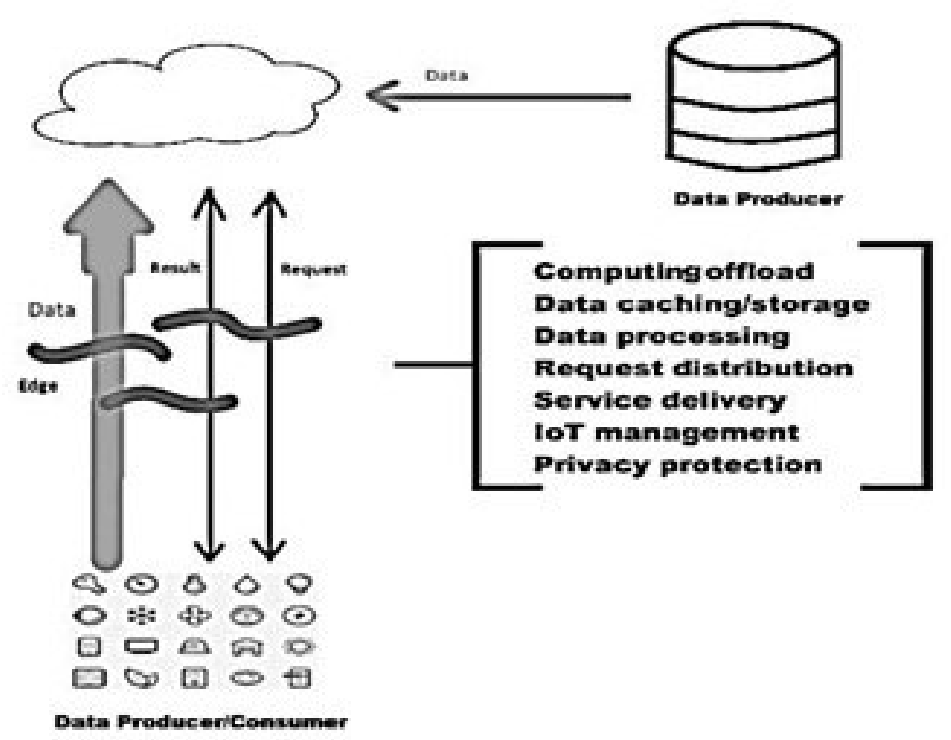

FIG. 4.1. Edge computing architecture.

5. System Architecture, Fog and Edge computing. Figure 5.1 exhibits a complete diagram of a Health-IoT system that shows how the elements can be systemized in such a scattered way over the three layers which can be used in smart hospitals. Among these classifications, the implanted sensors note the associated material of the patient's health, thus the private monitoring of various variables can be furnished by helping the patient. This health data can be also boosted by means of date, time, location, temperature, etc. Circumstanceawareness allows identifying a typical design and creates more exact inferences regarding the situations. The staff like CAT scan, magnetic resonance imaging can be attached with other medical equipment for transferring information from machine. The machine construction contains following important elements:

- Medical Sensors and Actuators networks: Some omnipresent identification, sensing, and transmission capability, biomedical and perspective signals are allowed to betaken from the body and surrounding. Some wireless or wired communication protocols such as Bluetooth, Wi-Fi, networks of intelligent e-Health helps in transferring the data to the access.

- Gateways: After several geographically scattered intelligent e-Health gateways this layer is constructed, such as developing a virtual fog. Every gateway, acts as a connection point among a sensor network along with the local switch/ Internet which helps in different transmission protocols. These higher-level facilities like data aggregation, filtering and dimensionality depletion can only be accepted if data from various subnetworks, bearings protocol conversion provided to them.

- Back-End System: A cloud computing programme that executes streaming, data warehouse as well as data analytic is achieved in this Back-end scheme. At last, it can adjunct the web client exhibition such as GUI regarding final picture and feedback. A source of large data [36, 37, 63] for statistical and epidemiological detecting forthcoming epidemic diseases can be constituted by the possessed health and setting data.

5.1. Properties in addition to features of the smart e-Health gateways. To help several wireless proprieties and take charge of inter-device transmission are the most significant part of this gateway. According to this part, we enlarge its particular part which turns out to be fog enabler by (i) establishing the orchestra by the linkage of gateways and (ii) applying various characteristics like acting as repository to volatile store users' data with sensors' and combining them with aggregation,interpretation techniques, and data fusion. For the local pre-processing of sensors' data these techniques are essential and can be called as an intelligent e-Health Gateway. 


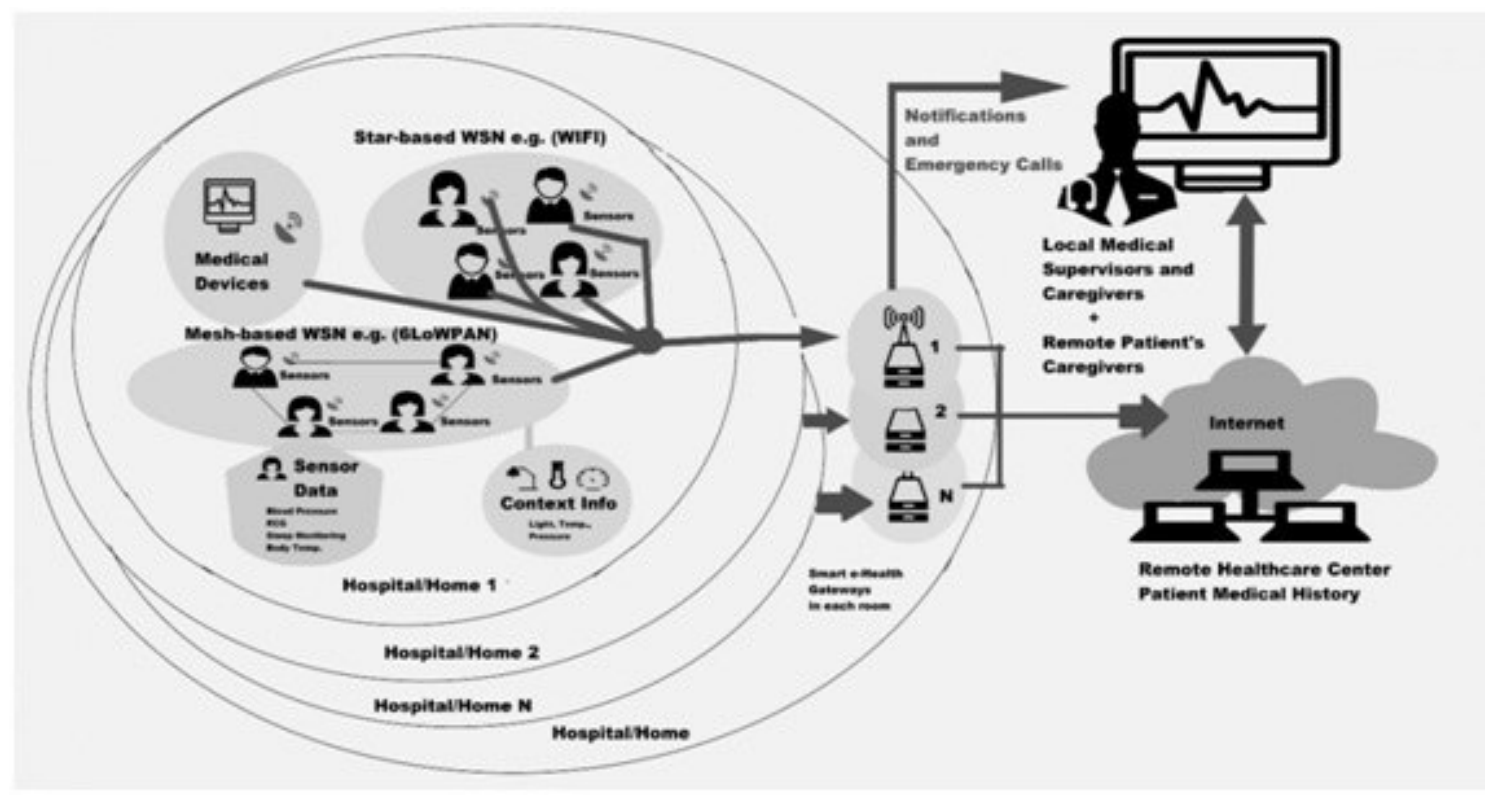

FIG. 5.1. Smart e-health monitoring system

5.1.1. Local data processing. Between the several characterised fogs computing has further added edge of local data handling which is executed to give smartness by which the streaming data is surveyed locally at the gateway. Agreeing towards the architecture of the machine, fog layer requirements constant approach of a huge quantity of sensory data in less time and react properly in surrounding to several situations. This task develops to be more vital in medical cases by allowing the machine to response as quickly as it can in case of medical crisis [38, 61]. Figure 5.2 explains the usage of a local processing unit for data filtering, data compression, data fusion, and data analysis with a conceptual architecture of an intelligent gateway.

5.1.2. Data filtering. Accepting data,after number of sensing devices and making it important to use it properly, early bird processes at the edge previous doing some kind of high-tech processing example data survey is really important. Bio-signals sucked up from any patient are always major sources of data for evaluating a patient condition. These generally have complex figures with little voltages and lots-off frequencies. Throughout the time of gathering information from a patient body, sound is also collected to the bio-signals and twists the indication standard. Such disturbances in the electromagnetic interference from other electrical machines, electric power grid, and unsuitable extension of sensors to patient's body can be built by several resources like oscillations of ac. These problems can be directed by the smart e-Health gateway at the fog layer in which it interfaces sensors at straight. By the use of several transmission protocols the fog layer accepts digitalised signals from sensors. Even though sensors might be executing light-pressures training to eradicate disturbances to a specific standard at the data receiving unit, additional tough and complicated information filtering at the fog layer is of primary importance.

5.1.3. Data compression. To minimize transmission waiting and power disbursed throughout transaction; the factors of data transmission, data compression is very essential. Both lossy and lossless data subjected on the solicitation are squeezed broadly utilizing it in the IoT domain. Computation for executing composite algorithms more demand than the Lossless data compressed for its short coming. So, for lossless data compression methods are advanced which have the ability and necessities for a processor speed and memory size. Both lossy and lossless compression technique are appropriate in the system layer of Health-IoT machine. Although, some restrictions occurs just as battery life time and availability of treating energy in some cases where lossy data compressing technique is more suitable for resource-constrained sensors. For example, numerous standard lossless ECG looseness techniques [39-40] don't suitable to many different kind of sensors like in lossy com- 


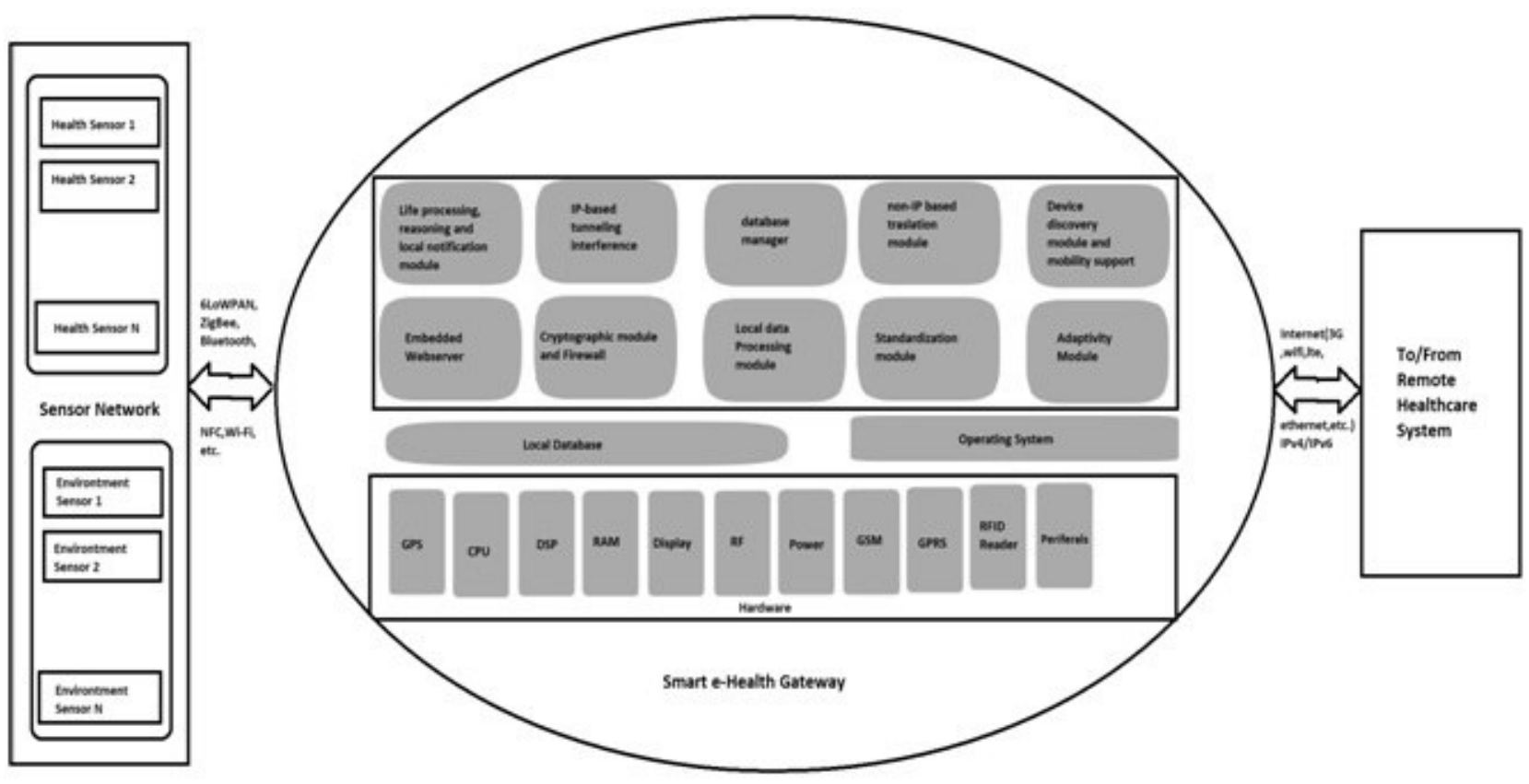

FIG. 5.2. Gateway architecture of smart e-health system.

pression techniques, for instance the technique proposed by $\mathrm{Yu}$ et al. [41], were practical in hardware demand with its features. For checking all structures of the signals are remained ensure of with big accuracy which is presented as in real-time ECG monitoring, has an advantage to have lossless compression. By discharging the liability from system layer Fog computing supply the required computational energy for systematic directed towards lossless data compression algorithms with complexities. While consuming lossless data compressing techniques it can authorize real-time actions.

5.1.4. Data blending. Data blending authorizes to productively lower the capacity of data, and there by lower the energy necessary for transmitting data. Whereas, Data fusion is actually divided in three different parts: cooperative,complementary as well as competitive [42]. Corresponding data fusion is applied on the fog layer to obtain stronger worldwide knowledge. Gathering the temperature dissimilarity between body and the neighbouring at any occasion is given from two different sensors. Competitive data fusion is also employed on the edge that data since a distinguished factor is gathered from various resources to refine the precision and the stability of outcomes in instances of sensors' collapses. Ultimately, collaborative data fusion too gives advantages in the edge such that fresh data is gathered in intelligent gateways from the diverse information which is gathered from different origins. For example, cooperative data fusion provides all-inclusive information regarding the medical condition of a patient from their important symptoms.

5.1.5. Data analysis. The reactivity of the machine can be enhanced by implementing localized data examination in the edge. It may help the machine to erase and forecast alarming conditions. For example, detecting the fall of an aged person, the fog layer could provincially supply detection of falling of a person regards giving out not only despatching variables to a cloud and resting for the replies. Subsequently, the machine responds to the alarming condition as fast as possible and is more trust worthy and executes real-time reactions. In the fog layer,Apart from that the sensitivity of the machine, exploiting facts examination to allow the machine on the way to lower the latencies along with crucial variables to a maximum limit. Moreover, localized data examination and local response from the sensory information upgrade the machine dependability and stability in case of non-avail ability of Internet connectivity. For continuing distant observing of discrete suffering from persist entitles, Internet disconnection can be recurrent happenings. Fog computing authorizes to remain all the performance of the machine function close by. Additionally,giving out findings in a localized 
repository in the fog layer as well as feasible to rescue the sensory information after harmonizing them along with the Cloud and connecting it later when it is back in position and in operation.

5.1.6. Adaptability. Taking into account, the implementation of fog layer in different situation, some early defined variables (example:sensors to cloud transmission rate) are fixed considering the use case. Nevertheless, this is very important for the fog layer to be customizable as well as adaptable over time, exceptionally when crucial incident happens. The customization may be vigorously implemented for different assistances employing progressive ML algorithms like incremental SVM and incremental NN. Data transmission of the fog layer requires being regularly incremented, which combines data appeal from the sensors and also transmitting the data taking it as a ratio of the cloud to the fog layer. For example, on continuing observation of a sick person sustaining cardiovascular disease, the machine must acquire to grow the appeal rates (priority) for heart-related variables on identifying unusual heart-related issues. Nevertheless, rate of transmission to the cloud is executed by allocating prime concerns to various services besides parameters. The prime concerned of rate of the cloud for patients to the transmission of data with critical illness involves is being elevated, while low transmission rate is required for the patients suffering from chronic diseases. As a conclusion,the performance of machine is not good as the adaptive fog computing thereby growing sensitiveness with explicitness of crucial limitations and on dependability of the prime concern the system adapts.

5.1.7. Local storage. To safeguard that the machine is able to recuperate evenly, the data, gateways must be efficient of keeping the arriving data with the local drive. The OS on the gateway manages the local repository and also keeping the data inside of a non-volatile storage. Data can be kept in local drive in a squeezed or protected way depending on the category as well as consequences. Data can be transported into a medical quality formats like Health Level-7 (HL7) [43] if needed in the repository. For additional purposes of the gateway storing of data is correspondingly important. As considered before, access will be in charge of data survey, compressing, filtration as well as encoding; all these tasks may need a local volatile drive. The pace for transmitting data from the gateway to the cloud is restricted by network transmission capacity, besides computations were restricted by giving out capacity of the gateway, for the situation of inequality of data processing and data transmission, constant data drift along with the local drive will act as a cache to appliance uniformity. When the network is not available but it makes the Data drive on gateways machine dependable and powerful. The local repository is managed by the database administrative unit shown in Figure 5.2.

5.1.8. Local impulsion. The actuation can be categorized into different configurations in the IoT-based healthcare system. The actuation can be used in the configuration of data streaming, administrating medical actuators as well as sensor network reconfiguration. , A foreseeable and fast reaction time is proven in many situations. Illustrations for fast reaction actuators modify the frequency of electrical nerve which is restored depending on the heart rate, otherwise altering insulin liberate rate in unmanned pumps based on the additional key indications and patient blood Glucose. In real-time to regulate panel for medical exception lists is important for transmission to slow down where at least samples/second rate is required to attain when there is a streaming in a patient's medical signals. Utilizing the networking potential along with local processing force the gateways are capable of streaming real-time signals like ECG and PPG (photo plethysmogram) towards a user machine (i.e., tablet), on the Internet connectivity separately. For intelligent e-Health gateways at the edge of the network some statements are required between the notable functions. Health care observing systems frequently requires notifying and informing care givers, medical teams and the patient about censorious circumstances that need immediate response. Any miscarriage in the notification utility may cause critical issues for medical therapy as well as the patient. Differentiate of sending notifications to a cloud server which is capable after numerous practices, a gateway which has restricted measures only can inform through few fix media. However, there maybe a lack of cloud server but the gateway-based statements proceed autonomously (e.g., via the local grid system or GSM) throughout the server, after amplifying the constancy of the machine we must be sure that users can obtain important reports in time.

5.1.9. Security. In the Health-IoT systems security is regarded as of great importance on the morale that in secured machine may have dangerous problems. In Linux the gateways like the IP table is exploited when it is approached by a big level of security as well as operating system level. When Linux kernel firewall supplies IP tables and IP FW it is also applied for the set of regulations in the network packets configuring the IP packet 
table. At some ports IP tables are constructed for giving privileges to communicate while other ports will be obstructed meant for obstructs dispensable traffic $[44,60]$. Although the gateway behaves as an embedded web server when the set-up is not available or when it is required, it may convey through secured validate sensor junctions plus HTTP sustaining the integrity,privacy, and originality of the machine. Even though IP tables give a few lead, they can' be reviewed as a powerful appliance for safety. In structured to have a towering level of safety, IP tables need to be in alliance with extra powerful safety methods. To label these problems, distinct advance towards have been currently suggested in the compositions [45-47, 59]. Although, cryptanalysis performance in these advancements are bulky because of the capability of force and processing capability limits building these illogical for resource-limited system. In a current report cantered on safety, Rahimi et al. [48, 49] proposed a safe and robust authenticated and authorized proposal for the Health-IoT machines which is required for few processing power in the edge. More accurately, on the conflict to run safe techniques at resource-limited sensor nodes, and the proposal utilizes features of an intelligent gateway in the fog computing for high end security which is related to the workload.

5.1.10. Inter operability and re-configurability. Apart from the uniformities attempts, it was apparent that inter operability has a crucial part for the accomplishment of Health-IoT tools. By alike heterogeneous mixture of grid system technologies, platform and protocol selection for executing IoT-based framework, implementing these approach is an apparent confrontation. This intelligent e-Health gateway has a crucial part forgiving inter operability for different sensors attached through distinguished grid system edges. As depicted in Figure 5.2, the intelligent e-Health Gateway utilising either wireless network or wired connections are attached to the context sensors plus the health sensors where it implements various grades (e.g., Wi-Fi, ZigBee, Bluetooth, $6 \mathrm{LoWPAN}$, ) to interact to the gateway. The cleverness of the gateway arises within the shape of uncomplicated combination of technologies and standards with these heterogeneous network protocols, soit permits them to interchange data along with effort easily.

Technical aspect of Inter operability. The different machine parts in the IoT-based machine are developed by number of sellers, and thereby they implement various grid system standards and interfaces. In this intelligent e-Health gateway development, technical inter operability can be gained by instructing switched data to the intelligent e-Health gateway that has various interfaces.

Syntactic Inter operability. The layer for Syntactic inter operability actually depends on the foregoing, technical inter operability layer. This handles the structure of messages interchanged across the systems.

Semantic Inter operability. To give semantic inter operability in two different types a proposed intelligent eHealth gateway had been developed. The first inter operability represents the information gathered from sensor nodes when it is concerned with additional systems. On the other hand,Internet plus the human readable data is attached with the gateway.

5.1.11. Discovering devices and mobility assistance. Usually mobility has two major techniques, handover and roaming, those are required for loss of data and interruption in services, and to manage the Quality of Service (QoS). In mobile host, bestowal happens when the connection channel is switched to other channel during roam occurs while going from one network to other. Macro and micro class are two divisions of Mobility and they were expressed as mobility inside the various network regions also inside the network region, commonly [48, 57]. There remain a number off ewer techniques which hold up mobility in edge routers [50, $56,58]$. Nevertheless, substitute technique is not present in this period to wholly represent the toughest the IoT scenario. For instance, inconsistency with routing which is multi hop, besides the need of NS (Neighbour Solicitation)/NA (Neighbour Advertisement) interchanges occur among the dualistic real problems inside the proxy IPv6 mobile. NEMO changes to further complex when various kinds of mobility (micro and macro) happen at a time.

5.1.12. Energy efficient criteria for sensor nodes. Recently, several approaches [51, 54, 55, 62] are being focused on giving energy efficient for IoT based Health products. When Gia et al. [53] was utilizing the short power transmission protocol, Otto et al. [52] executed the authentic signal handling on sensor nodes towards the sensor nodes conserving the energy of transmission. The appreciable quantity of energy can be easily stored or kept unused through fog computing by redistributing a few capacities from sensor module to intelligent gateways.However,those approaches may increase the energy efficiency of the sensor nodes. 
6. Conclusion. The research work presented in this article focuses on retrospection of Edge and Fog computing applications in healthcare. These jargons are associated with Cloud computing and named according to their architectural relationship. Various applications can function more effectively using Edge and Fog level rather than migrating to the Cloud. This is for various factors like nature of client, data locality, grid system overhead, device and cloud resources and their availability etc. The main intention remains to make the service available in all types of circumstances. It has been found that various healthcare related applications are more applicable for execution in Edge and Fog rather than Cloud for its solution to the various constraints of the various sensors. However, the review depicts that a massive computation which happens in healthcare applications need to be processed in a layer that exist between the sensor network and the cloud. The articles reviewed here also show that all the network layers have potential to get involved in computation work. Apart from this, we also have highlighted the trade-off when allocating computational task to the levels of the network and discussed the challenges and security issues of fog and edge computing related to healthcare applications. Though the sensor devices are not powerful enough for performing the computation task, they overcome this limitation by offloading the computation task. In addition, due to some restrictions, cloud computing is not an efficient solution for such offloading. But, the flexibility of fog computing allows to induct computation as a part of the network infrastructure, therefore regarded as a most appropriate solution for healthcare.

\section{REFERENCES}

[1] J. BurT, "Fog computing aims to reduce processingburden of cloud systems," http://www.eweek.com/grid systeming/fogcomputing-aims-to-reduce-processing-burden-of-cloud-systems, html, 2010.

[2] Health Level Seven Int'l. Introduction to HL7 Standards, 2012, www.hl7.org/implement/standards [accessed 2015-07-30].

[3] M.L. Hilton. Wavelet and wavelet packet compression of electrocardiograms. IEEE Trans.Biomed., 44(5):394 - 402, 1997.

[4] Z. Lu, D. Youn Kim, and W.A. Pearlman. Wavelet compression of ECG signals by the setpartitioning in hierarchical trees algorithm. IEEE Trans. Biomed., 47(7):849 - 856, 2000.

[5] R. Benzid, A. Messaoudi, And A. BoussaAd, "Constrained ECG compression algorithmusingthe block-based discrete cosine transform. Digital Signal Processing", 18(1):56 - 64, 2008.

[6] H. F. Durrant-Whyte. Sensor models and multisensor integration. Int. J. Rob. Res., 7(6):97-113, 1988.

[7] T. Nguyen Gia, T. Igor, V.K. Sarker, A.-M. Rahmani, T. Westerlund, P. Liljeberg, and H.Tenhunen. Iot-based fall detection system with energyefficient sensor nodes. In IEEE Nordic Circuits and Systems Conference (NORCAS'16), 2016.

[8] T. Nguyen Gia, M. Jiang, A.-M. Rahmani, T. Westerlund, K. Mankodiya, P. Liljeberg, and H.Tenhunen. Fog computing in body sensor grid systems: An energy efficient approach. In IEEE International Body Sensor Grid systems Conference (BSN'15),2015.

[9] T. Nguyen Gia, M. Jiang, A. M. Rahmani, T. Westerlund, P. Liljeberg, and H. Tenhunen. Fog computing in healthcare internet of things: A case study on ecg feature extraction. In Proceeding of International Conference on Computer and Information Technology, pages 356-363,2015.

[10] B. Negash, A. M. Rahmani, T. Westerlund, P. Liljeberg, and H. Tenhunen. Lisa:Lightweight internet of things service bus architecture. Procedia Computer Science, 52:436 - 443,2015. The 6th International Conference on Ambient Systems, Grid systems and Technologies (ANT-2015), the 5th International Conference on Sustainable EnergyInformation Technology (SEIT-2015).

[11] N. Paul, T. Kohno, And D. C. Klonoff. A review of the security of insulin pumpinfusionsystems. Journal of Diabetes Science and Technology, 5(6):1557-1562, 2011.

[12] netfilter/iptables - nftables project. http://netfilter.org/projects/nftables/ [accessed 2015-07-24].

[13] G. Kambourakiset, E. Klaoudatou, and S. Gritzalis. Securing Medical SensorEnvironments:TheCodeBlue Framework Case. In Proceeding of The Second International Conference on availability, Reliability and Security, pages 637-643, 2007.

[14] R. Chakravorty, A programmable Service Architecture for Mobile Medical Care, In Proceeding of Fourth Annual IEEE International Conference on Pervasive Computing and Communications Workshops, pages 5 - 536, 2006.

[15] J. Ko, J. H. Lim, Y. Chen, R. Musvaloiu-E, A. Terzis, G. M. Masson, T. Gao, W. Destler, L. Selavo, and R. P. Dutton. Medisn: Medical emergency detection in sensorgrid systems. ACMTrans. Embed. Comput. Syst., 10(1):11:1$11: 29,2010$.

[16] S.R. Moosavi, T.N. Gia, A. Rahmani, E. Nigussie, S. Virtanen, H. Tenhunen, And J. Isoaho. SEA: A Secure and Efficient Authentication and Authorization Architecture for IoT-Based Healthcare Using Smart Gateways. In Proceeding of 6th International Conference on AmbientSystems, Grid systems and Technologies, page 452-459, 2015.

[17] S.R. Moosavi, T.N. Gia, E. Nigussie, A. Rahmani, S. Virtanen, H. Tenhunen, and J. Isoaho, Session Resumption-Based End-to-End Security for Healthcare Internet-of-Things, In Proceedingof IEEE International Conference on Computer and Information Technology, pages581-588,2015.

[18] Y. Chen, W. Shen, H. HuO, And Y. Xu, A smart gateway for health caresystem using wireless sensor grid system, in 2010 Fourth International Conference on Sensor Technologies and Applications (SENSORCOMM), July 2010, pp. 545-550.

[19] S. Mohapatra and K. S. Rekha, Sensor-cloud: a hybrid framework forremote patient monitoring, Int. J. Comput. Appl, 
vol. 55, no. 2, pp.1-11, 2012.

[20] C. O. Rolim, F. L. Koch, C. B. Westphall, J. Werner, A. Fracalossi, and G. S.Salvador, A cloud computing solution for patient's datacollection in health careinstitutions, in ETELEMED'10, Second International Conference on e-Health, Telemedicine, and Social Medicine,2010. IEEE, 2010, pp. 95-99.

[21] S. Yang And M. Gerla, Personal gateway in mobile health monitoring, in 2011 IEEE International Conference on Pervasive Computing and Communications Workshops (PERCOM Workshops), March 2011, pp. 636-641.

[22] J. W. Yoon, Y. K. Ku, C. S. NAm, And D. R. Shin, Sensor grid systemmiddlewarefor distributed and heterogeneous environments, in NISS'09.International Conference on New Trends in Information and ServiceScience, 2009. IEEE, 2009, pp. 979-982.

[23] W. Shen, Y. Xu, D. Xie, T. Zhang, and A. Johansson, Smart borderroutersfore-healthcare wireless sensor grid systems, in 2011 7th International Conference on Wireless Communications, Grid system and MobileComputing (WiCOM), Sept 2011, pp. 1-4.

[24] T. H. Laine, C. LeE, AND H. Suk, Mobile gateway for ubiquitous healthcare system using zigbee and Bluetooth, in 2014 Eighth InternationalConference on Innovative Mobile and Internet Services in UbiquitousComputing (IMIS). IEEE, 2014, pp. 139-145.

[25] A Phone-centred body sensor grid system platform: Cost, energy efficiency and user interface. Institute of Electrical and Electronics Engineers,Inc., April 2006. [Online]. Available: http://research.microsoft. com/apps/pubs/default.aspx? id $=103003$.

[26] K. PARK And J. PAK, An integrated gateway for various P.hds in uhealthcareenvironments, BioMed Research International, 2012.

[27] A. M. Rahmani, N. K. Thanigaivelan, T. N. Gia, J. Granados, B. Negash, P. Liljeberg, and H. Tenhunen, Smart ehealth gateway: Bringing intelligenceto internet-of-things based ubiquitous healthcare systems, in Annual IEEE Consumer Communications and Grid systemConference. IEEE, 2015, pp. 826-834.

[28] F. Bonomi, R. Milito, P. Natarajan, and J. Zhu, Fog computing: Aplatformfor internet of things and analytics, in Big Data and Internetof Things: A Roadmap for Smart Environments. Springer, 2014, pp.169-186.

[29] N. Bessis And C. Dobre, Big data and internet of things: a roadmap forsmart environments. Springer, 2014.

[30] F. Bonomi, R. Milito, J. Zhu, and S. Addepalli, Fog computing and itsrole in the internet of things, in Proceedings of the first edition of the MCC workshop on Mobile cloud computing. ACM, 2012, pp. 13-16.

[31] M. Satyanarayanan, P. Bahl, R. Caceres, and N. Davies, The case forVM-based cloudlets in mobile computing, IEEE Pervasive Comput.,vol. 8, no. 4, pp. 14-23, Oct./Dec. 2009.

[32] OpenFog Architecture Overview. OpenFogConsortiumArchitecture Working Group. Accessed on Dec. 7, 2016.[Online]. Available: http://www.openfogconsortium.org/wp-content/uploads/OpenFog-Architecture-Overview-WP-2-2016.pdf.

[33] K. Ha et AL., Towards wearable cognitive assistance, in Proc. 12th Annu. Int. Conf. Mobile Syst. Appl. Services, Bretton Woods, NH, USA,2014, pp. 68-81.

[34] B.-G. Chun, S. Ihm, P. Maniatis, M. Naik, and A. Patti, Clone Cloud: Elastic execution between mobile device and cloud, in Proc. 6th Conf. Comput. Syst., Salzburg, Austria,2011, pp. 301-314.

[35] Atta-ur-Rahman, J. Alhiyafi, Health Level Seven Generic Web Interface", J. Comput. Theor. Nanosci. 15 (4), pp. 1261$1274,2018$.

[36] D. LANey, 3D Data Management: Controlling Data Volume, Velocity, and Variety, Technical Report, Application Delivery Strategies by META Group Inc., 2001.

[37] M. Beyer, Solving Big Data Challenge Involves More Than Just managing Volumes of Data, 2016. http://www.gartner.com/newsroom/id/ 1731916.

[38] J. Granados, A.M. Rahmani, P. Nikander, P. Liljeberg, H. Tenhunen, Towards energy-efficient HealthCare: An internetof-things architecture using intelligent gateways, in: Proc. of International Conference on Wireless Mobile Communication and Healthcare, 2014, pp. 279-282.

[39] M.L. Hilton, Wavelet and wavelet packet compression of electrocardiograms, IEEE Trans. Biomed. 44 (5) (1997) $394-402$.

[40] Z. Lu, D. Youn Kim, W.A. Pearlman, Wavelet compression of ECG signals by the set partitioning in hierarchical trees algorithm, IEEE Trans. Biomed. 47 (7) (2000) 849-856.

[41] R. Benzid, A. Messaoudi, A. BoussaAd, Constrained ECG compression algorithm using the block-based discrete cosine transform, Digit. Signal Process. 18 (1) (2008) 56-64.

[42] F. Tounti, R. TABish, U-healthcare system: State-of-the-art review and challenges, J. Med. Syst. 37 (3) (2013).

[43] H.F. Durrant-Whyte, Sensor models and multisensor integration, Int. J. Robot. Res. 7 (6) (1988) 97-113.

[44] Health Level Seven Int'l, Introduction to HL7 Standards, 2017,www.hl7.org/ implement/standards. (Accessed on 30 July 2017).

[45] Netfilter/iptables -nftables project, http://netfilter.org/projects/nftables/. (Accessed on 24 July 2015).

[46] G. Kambourakiset, E. Klaoudatou, S. Gritzalis, Securing medical sensor environments: The codeblue framework case, in Proceeding of the Second International Conference on Availability, Reliability and Security, 2007, pp. 637-643.

[47] J. Ko, J.H. Lim, Y. Chen, R. Musvaloiu-E, A. Terzis, G.M. Masson, T. Gao, W. Destler, L. Selavo, R.P. Dutton, MEDiSN: medical emergency detection in sensor grid systems, ACM Trans. Embed. Comput. Syst. 10 (1) (2010) 11:111:29.

[48] S.R. Moosavi, T.N. Gia, A. Rahmani, E. Nigussie, S. Virtanen, H. Tenhunen, J. Isoaho, SeA: A secure and efficient authentication and authorization architecture forIoT-based healthcare using smart gateways, in: Proceeding of 6 th International Conference on Amb ient Systems, Grid systems and Technologies, 2015, pp. 452-459.

[49] S.R. Moosavi, T.N. Gia, E. Nigussie, A. Rahmani, S. Virtanen, H. Tenhunen, J. Isoaho, Session resumption-based end-to-end security for healthcare internet of-things, in: Proceeding of IEEE International Conference on Computer and 
Information Technology, 2015, pp. 581-588.

[50] Z. Shelby, C. Bormann, 6LoWPAN: The Wireless Embedded Internet, Wiley, UK, 2009.

[51] S. Gundavelli, K. Leung, V. Devarapalli, K. Chowdhury, B. Patil, Proxy Mobile 2 IPv6, Internet Engineering Task Force, 2008.

[52] C. Отto, A. Milenkovi?, C. Sanders, E. Jovanov, System architecture of a wireless body area sensor grid systemfor ubiquitous health monitoring, J. Mob. Multimedia 1 (4) (2006) 307-326.

[53] T. Nguyen Gia, N.K Thanigaivelan, A.M. Rahmani, T. Westerlund, P. Liljeberg, H. Tenhunen, Customizing 6LoWPAN grid systems towards Internet-of-Things based ubiquitous healthcare systems, in: Proceeding of NORCHIP, 2014, pp. 1-6.

[54] Kumari., S. Tanwar., S. Tyagi, N. Kumar, M. Mansberg, K. K. R. Choo, Multimedia Big Data Computing and Internet of Things Applications: A Taxonomy and Process Model, Journal of Network and Computer Applications, 124:169-195, 2018.

[55] Kumari A., Tanwar S., Tyagi S., Kumar N., Fog Computing for Healthcare 4.0 Environment: Opportunities and Challenges, Computers and Electrical Engineering, Volume 72, 2018, Pages 1-13 .

[56] J. Vora, P. Devmurari, S. Tanwar, S. Tyagi, N. Kumar, M. S. Obaidat, Blind Signatures Based Secured E-Healthcare System, International Conference on Computer, Information and Telecommunication Systems (IEEE CITS-2018), Colmar, France, 11-13 July 2018, pp. 177-181

[57] J. Vora, P. Italiya, S. Tanwar, S. Tyagi, N. Kumar, M. S. Obaidat, and K-F. Hsiao, Ensuring Privacy and Security in EHealth Records, International Conference on Computer, Information and Telecommunication Systems (IEEE CITS-2018), Colmar, France, 11-13 July 2018, pp. 192-196

[58] S. Tanwar, P. Patel, K. Patel, S. Tyagi, N. Kumar, M. S. Obaidat, An Advanced Internet of Thing based Security Alert System for Smart Home, International Conference on Computer, Information and Telecommunication Systems (IEEE CITS-2017), Dalian University, Dalian, China, 21-23 July 2017, pp. 25-29.

[59] S. Tanwar, Tyagi S and Kumar S, The Role of Internet of Things and Smart Grid for the Development of a Smart City, Intelligent Communication and Computational Technologies (Lecture Notes in Networks and Systems: Proceedings of Internet of Things for Technological Development, IoT4TD 2017, Springer International Publishing, vol 19, pp. 23-33.

[60] A. Rahman, S Dash, M Kamaleldin, A Abed, A Alshaikhhussain, H Motawei, N Al. Amoudi, W Abahussain, A Comprehensive study of mobile computing in Telemedicine, A. K. Luhachet. al. (Eds):ICAICR 2018,CCIS 956, pp. 413425, 2019. Springer, Singapore

[61] A Rehman, S Dash, K Sultan, MA Khan, Management of Resource Usage in Mobile Cloud Computing, International Journal of Pure and AppliedMathematics, Volume 119 No. 16, 2018, 255-261.

[62] A. Solanki A., Nayyar A. Green Internet of Things (G-IoT): ICT Technologies, Principles, Applications, Projects, and Challenges. In Handbook of Research on Big Data and the IoT (pp. 379-405). IGI Global, 2019

[63] S.P. Singh, Nayyar, A., Kumar, R., Sharma, A. Fog computing: from architecture to edge computing and big data processing. The Journal of Supercomputing, 1-36, 2018

[64] S. Yi, Z. HaO, Z. QIn, AND Q. LI, Fog computing: Platform andapplications, in Proc. 3rd IEEE Workshop Hot Topics Web Syst.Technol. (HotWeb), Washington, DC,USA, 2015, pp. 73-78.

Edited by: Anand Nayyar

Received: Jan 9, 2019

Accepted: Mar 17, 2019 